\title{
A new quasicrystal approximant in the Sc-Pd system: from topological data mining to the bench
}

\author{
Pavlo Solokha ${ }^{1 *}$, Roman A. Eremin ${ }^{2,3}$, Tilmann Leisegang ${ }^{2,4}$, Davide M. Proserpio ${ }^{2,5}$, \\ Tatiana Akhmetshina $^{2,3}$, Albina Gurskaya ${ }^{2,3}$, Adriana Saccone ${ }^{1}$, Serena De Negri ${ }^{1}$ \\ 1 Università degli Studi di Genova, Dipartimento di Chimica e Chimica Industriale, \\ Via Dodecaneso 31, I-16146 Genova, Italy \\ 2 Samara Center for Theoretical Materials Science (SCTMS), Samara State Technical University, \\ Molodogvardeyskaya St. 244, Samara 443100, Russia \\ ${ }^{3}$ Samara Center for Theoretical Materials Science (SCTMS), Samara University, Ac. Pavlov St. 1, \\ Samara 443011, Russia \\ ${ }^{4}$ Institute of Experimental Physics, TU Bergakademie Freiberg, Leipziger Str. 23, 09599 Freiberg, \\ Germany \\ ${ }^{5}$ Università degli Studi di Milano, Dipartimento di Chimica, Via Golgi 19, 20133 Milano, Italy
}

\section{SUPPLEMENTARY MATERIALS}




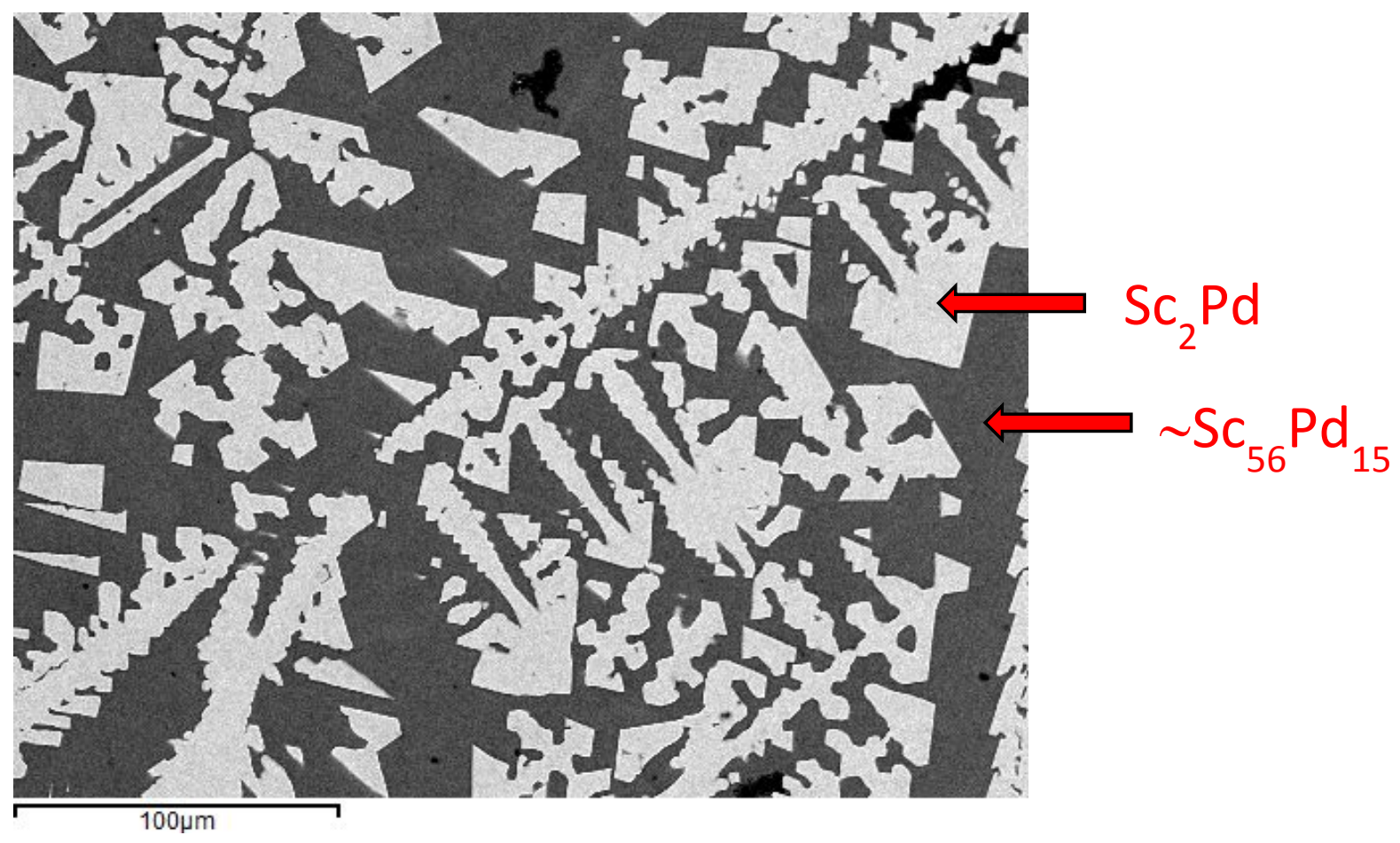

a)

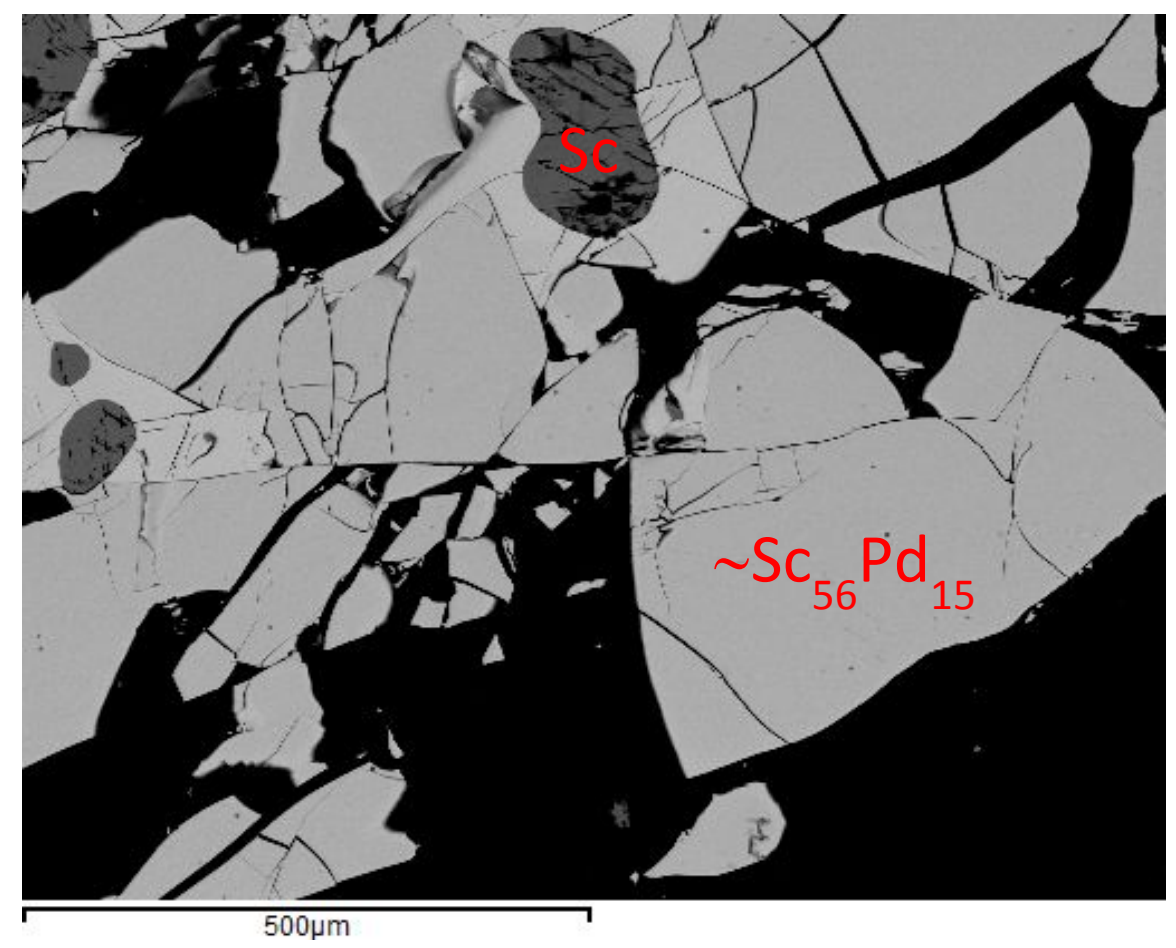

b)

Figure S1. Microphotographs (BSE mode) of the $\mathrm{Sc}_{74} \mathrm{Pd}_{26}$ (a) and $\mathrm{Sc}_{80} \mathrm{Pd}_{20}$ (b) alloys. Measured compositions: $\mathrm{Sc}_{2} \mathrm{Pd}: 68$ at. $\%$ Sc; $\sim \mathrm{Sc}_{56} \mathrm{Pd}_{15} 1 / 1 \mathrm{AC}: 78$ at.\% Sc. 


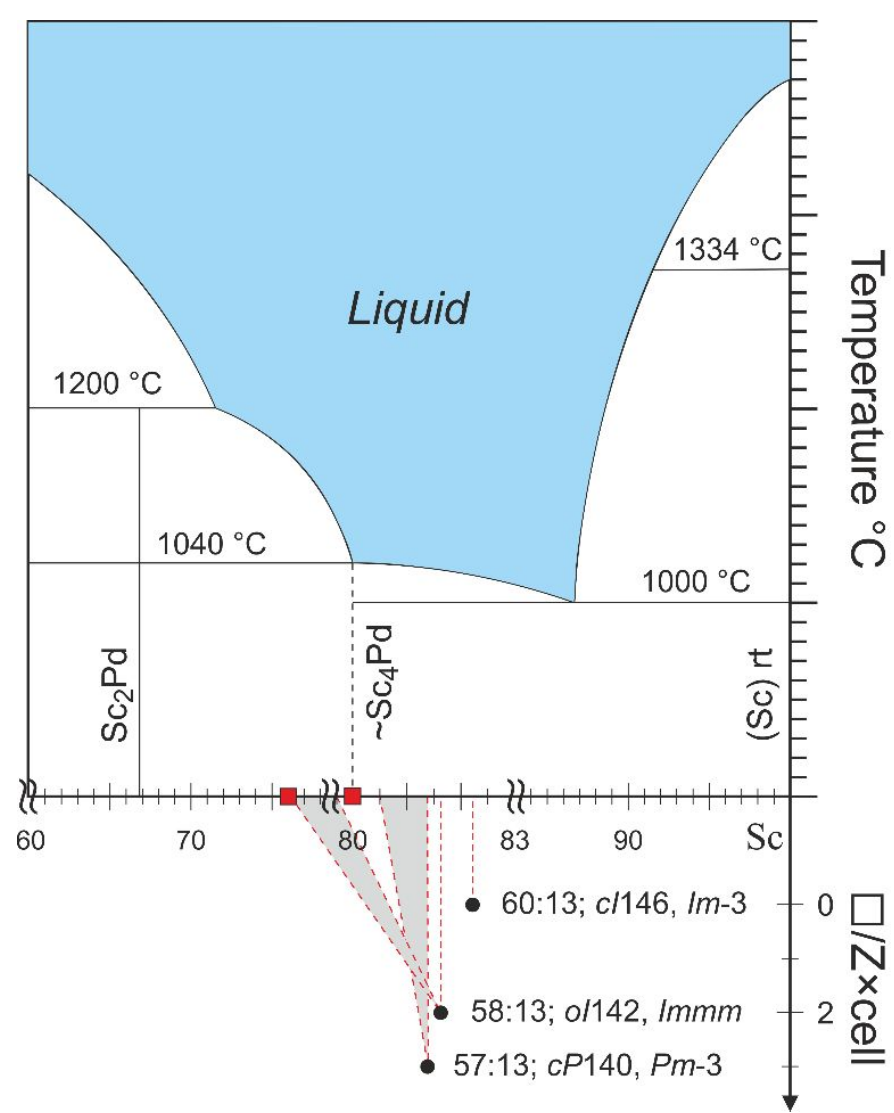

a)

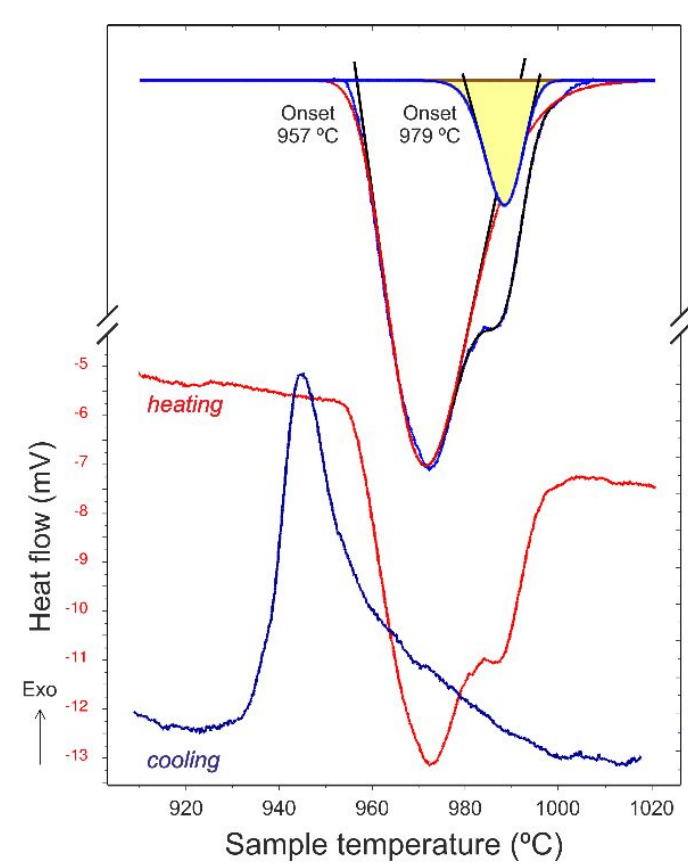

b)

Figure S2. a) Top: Sc-rich part of the Sc-Pd phase diagram taken from Okamoto². Bottom: the compositional distribution of binary Sc- $X 1 / 1$ ACs (where $X$ is late transitional element) containing Mackay cluster as a function of vacancy ( $($ ) concentrations $\square / Z \times$ cell; grey zones indicate the compositional shifts due to $\mathrm{Sc} / X$ substitution. Red squares correspond to $\mathrm{Sc}_{74} \mathrm{Pd}_{26}$ and $\mathrm{Sc}_{80} \mathrm{Pd}_{20}$ samples studied here.

b) DTA curve for the $\mathrm{Sc}_{80} \mathrm{Pd}_{20}$ alloy in the range $900-1020^{\circ} \mathrm{C}$. Red line is the heating curve and blue line - the cooling one. In the heating curve two partially overlapped thermal effects are distinguishable which were interpreted as the eutectic $\left(957^{\circ} \mathrm{C}\right)$ and the peritectic $(979$ ${ }^{\circ} \mathrm{C}$ ) effects reported in the literature at somewhat different temperatures. 
Table S1. Selected experimental and structure determination parameters for the two 1/1 ACs, crystals I and II, respectively. The standard deviations of the lattice parameters are given in parentheses.

\begin{tabular}{|c|c|c|}
\hline & Crystal I & Crystal II \\
\hline \multicolumn{3}{|c|}{ Independent spherical atom model (ISAM) refinement } \\
\hline Space group (№) & Immm (71) & $I m m m(71)$ \\
\hline Refined composition & $\mathrm{Sc}_{54.98} \mathrm{Pd}_{15.56}$ & $\mathrm{Sc}_{55.44} \mathrm{Pd}_{15.54}$ \\
\hline Pearson symbol, prototype & ol142-1.01, $\mathrm{Hf}_{54} \mathrm{Os}_{17}$ & $0 / 142-0.04, \mathrm{Hf}_{54} \mathrm{Os}_{17}$ \\
\hline Formula unit $Z$ & 2 & 2 \\
\hline$a(\AA)$ & $14.2681(7)$ & $14.2522(3)$ \\
\hline$b(\AA)$ & $14.3696(7)$ & $14.3725(3)$ \\
\hline$c(\AA)$ & $14.7848(7)$ & $14.7760(3)$ \\
\hline$V\left(\AA^{3}\right)$ & $3033.1(3)$ & $3026.7(10)$ \\
\hline Lin. absorption coefficient $\mu\left(\mathrm{mm}^{-1}\right)$ & 10.07 & 10.09 \\
\hline Unique refl./parameters/restrains & $2796 / 126 / 25$ & $2787 / 126 / 25$ \\
\hline GOF & 1.09 & 1.05 \\
\hline Independent reflections & 2550 & 2017 \\
\hline$R_{\mathrm{int}}$ & 0.0320 & 0.1067 \\
\hline$R_{1} / w R_{1}(I>2 \sigma(I))$ & $0.025 / 0.058$ & $0.037 / 0.069$ \\
\hline$R_{1} / w R_{1}$ (all data) & $0.028 / 0.029$ & $0.067 / 0.077$ \\
\hline$\Delta \rho_{\text {res }}(\max . / \min ).\left(\mathrm{e} / \AA^{3}\right)$ & $1.57 /-2.00$ & $1.32 /-1.50$ \\
\hline
\end{tabular}

Maximum Entropy Method (MEM) ED reconstruction

\begin{tabular}{l|l|l}
\hline Algorithm ${ }^{49}$ & Sakato-Sato & Sakato-Sato \\
Calculated composition & $\mathrm{Sc}_{54.6} \mathrm{Pd}_{15.1}$ & $\mathrm{Sc}_{54.4} \mathrm{Pd}_{15.2}$ \\
Grid (points) & $128 \times 128 \times 128$ & $128 \times 128 \times 128$ \\
Resolution $(\AA)$ & $0.11 \times 0.11 \times 0.11$ & $0.11 \times 0.11 \times 0.11$ \\
Prior structural model & $\mathrm{ISAM}, \mathrm{Sc}_{56} \mathrm{Pd}_{15}$ & $I \mathrm{SAM}, \mathrm{Sc}_{56} \mathrm{Pd}_{15}$ \\
Generalized $F$-constraint $n{ }^{46}$ & $(I m m m)$ & $(I m m m)$ \\
Static weighting 50 & 2 & 2 \\
$R_{1} / \mathrm{w} R_{1}(I>2 \sigma(I))$ & $H 0$ & $H 0$ \\
\hline
\end{tabular}


P. Solokha et al. A new 1/1 Mackay type approximant in the Sc-Pd system: from topological data mining to the bench. Supplementary Materials.

Table S2. Sc-containing Mackay-type q/r ACs. Space group symmetry, central atom (site), distribution of the elements within the three shells of Mackay cluster, underlying net topology of cluster assemblage, and ICSD code are listed.

\begin{tabular}{|c|c|c|c|c|c|c|c|c|}
\hline № & q/r-AC Composition & \multirow[t]{2}{*}{$\begin{array}{l}\text { Space } \\
\text { group }\end{array}$} & \multirow[t]{2}{*}{$\begin{array}{l}\text { Central atom } \\
\text { (Wyckoff site) }\end{array}$} & \multicolumn{3}{|c|}{$\begin{array}{l}\text { Mackay cluster } \\
1^{\text {st }} \text { shell; } 2^{\text {nd }} \text { shell; } 3^{\text {rd }} \\
\text { shell }\end{array}$} & \multirow[t]{2}{*}{$\begin{array}{l}\text { Net } \\
\text { topology }\end{array}$} & \multirow[t]{2}{*}{$\begin{array}{l}\text { ICSD } \\
\text { code }\end{array}$} \\
\hline \multicolumn{5}{|c|}{ binaries } & & & & \\
\hline 1 & 1/1-AC Sc ${ }_{56.8} \mathrm{Rh}_{13.6}$ & $\operatorname{Im} \overline{3}$ & $\operatorname{Rh}(2 a)$ & $\mathrm{Sc}_{12}$ & $\mathrm{Sc}_{30}$ & $\mathrm{Rh}_{12}$ & bcu & 105923 \\
\hline 2 & $1 / 1-\mathrm{AC} \mathrm{Sc}_{29} \mathrm{Fe}_{6}$ & $P m \overline{3}$ & $\mathrm{Sc}(1 a ; 1 b)$ & $\mathrm{Sc}_{12}$ & $\mathrm{Sc}_{30}$ & $\mathrm{Fe}_{12}$ & bcu & 103620 \\
\hline 3 & $1 / 1-A C \mathrm{Sc}_{57} \mathrm{Rh}_{13}$ & $\operatorname{Pm} \overline{3}$ & $\operatorname{Rh}(1 a ; 1 b)$ & $\mathrm{Sc}_{12}$ & $\mathrm{Sc}_{30}$ & $\mathrm{Rh}_{12}$ & bcu & 105922 \\
\hline 4 & $1 / 1-\mathrm{AC} \mathrm{Sc}_{57} \mathrm{Ru}_{13}$ & $P m \overline{3}$ & $\mathrm{Ru}(1 a ; 1 b)$ & $\mathrm{Sc}_{12}$ & $\mathrm{Sc}_{30}$ & $\mathrm{Ru}_{12}$ & bcu & 600484 \\
\hline 5 & $1 / 1-A C S c_{57} \mid r_{13}$ & $\operatorname{Pm} \overline{3}$ & $\operatorname{Ir}(1 a ; 1 b)$ & $\mathrm{Sc}_{12}$ & $\mathrm{Sc}_{30}$ & $\operatorname{Ir}_{12}$ & bcu & 600485 \\
\hline 6 & $1 / 1-\mathrm{AC} \mathrm{Sc}_{57} \mathrm{Pt}_{13}$ & $\operatorname{Pm} \overline{3}$ & $\mathrm{Pt}(1 a ; 1 b)$ & $\mathrm{Sc}_{12}$ & $\mathrm{Sc}_{30}$ & $\mathrm{Pt}_{12}$ & bcu & 600486 \\
\hline 7 & 1/1-AC Sc $c_{55.3} \mathrm{Au}_{15.7}$ & Immm & $\mathrm{Au}(2 a)$ & $\mathrm{Sc}_{12}$ & $\mathrm{Sc}_{30}$ & $\mathrm{Au}_{12}$ & bcu & 1407356 \\
\hline 8 & $2 / 1-\mathrm{AC} \mathrm{Sc}_{44} \mathrm{Os}_{7}$ & $F \overline{4} 3 m$ & Os $(16 e)$ & $\mathrm{Sc}_{12}$ & $\mathrm{Sc}_{30}$ & $\mathrm{Os}_{9} \mathrm{Sc}_{3}$ & pcu & 105565 \\
\hline 9 & $2 / 1-\mathrm{AC} \mathrm{Sc}_{44} \mathrm{Ir}_{7}$ & $F \overline{4} 3 m$ & $\operatorname{Ir}(16 e)$ & $\mathrm{Sc}_{12}$ & $\mathrm{Sc}_{30}$ & $\mathrm{Ir}_{9} \mathrm{Sc}_{3}$ & pcu & 640969 \\
\hline \multicolumn{9}{|c|}{ ternaries } \\
\hline 10 & $\begin{array}{l}1 / 1-\mathrm{AC} \\
\mathrm{Sc}_{11} \mathrm{Fe}_{1.536} \mathrm{Ga}_{1.232}\end{array}$ & Immm & Sc (2a) & $\mathrm{Sc}_{12}$ & $\mathrm{Sc}_{30}$ & $\mathrm{Ga}_{4} \mathrm{Fe}_{8}$ & bcu & 103461 \\
\hline 11 & $\begin{array}{l}\text { 1/1-AC } \\
\mathrm{Sc}_{13.41} \mathrm{Ga}_{1.42} \mathrm{Mn}_{2}\end{array}$ & Immm & Sc $(2 a)$ & $\mathrm{Sc}_{12}$ & $\mathrm{Sc}_{30}$ & $\mathrm{Ga}_{4} \mathrm{Mn}_{8}$ & bcu & 103809 \\
\hline 12 & $1 / 1-A C \mathrm{Sc}_{50} \mathrm{Rh}_{13.3} \mathrm{In}_{2.7}$ & $F m \overline{3}$ & $\mathrm{Rh}_{0.3} \ln _{0.7}(4 b)$ & $\mathrm{Sc}_{12}$ & $\mathrm{Sc}_{30}$ & $\mathrm{Rh}_{12}$ & fcu & 418501 \\
\hline 13 & $1 / 1-A C ~ S c_{50} \mathrm{Ir}_{13.6} \mathrm{In}_{2.4}$ & $F m \overline{3}$ & $\ln _{0.4} \mid \mathrm{r}_{0.6}(4 b)$ & $\mathrm{Sc}_{12}$ & $\mathrm{Sc}_{30}$ & $\operatorname{Ir}_{12}$ & fcu & 418502 \\
\hline 14 & $1 / 1-\mathrm{AC} \mathrm{Sc}_{100} \mathrm{Co}_{25} \mathrm{In}_{7}$ & $F m \overline{3}$ & $\ln (4 b)$ & $\mathrm{Sc}_{12}$ & $\mathrm{Sc}_{30}$ & $\mathrm{Co}_{12}$ & fcu & 259424 \\
\hline
\end{tabular}




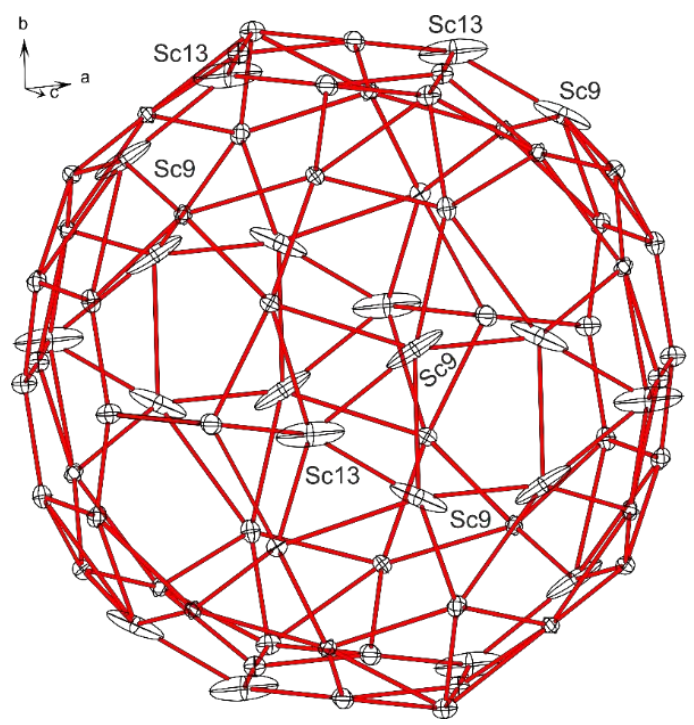

a)

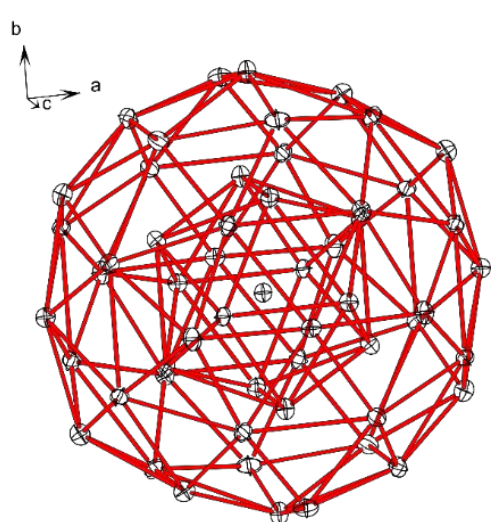

b)

Figure S3. Anisotropic displacement parameters for $\mathrm{Sc}_{56} \mathrm{Pd}_{15}$ ordered model shown with a $90 \%$ probability. a) ADPs for atoms belonging to the dRTH external shell; b) ADPs for the species belonging to the internal centered Mackay cluster of 55 atoms.

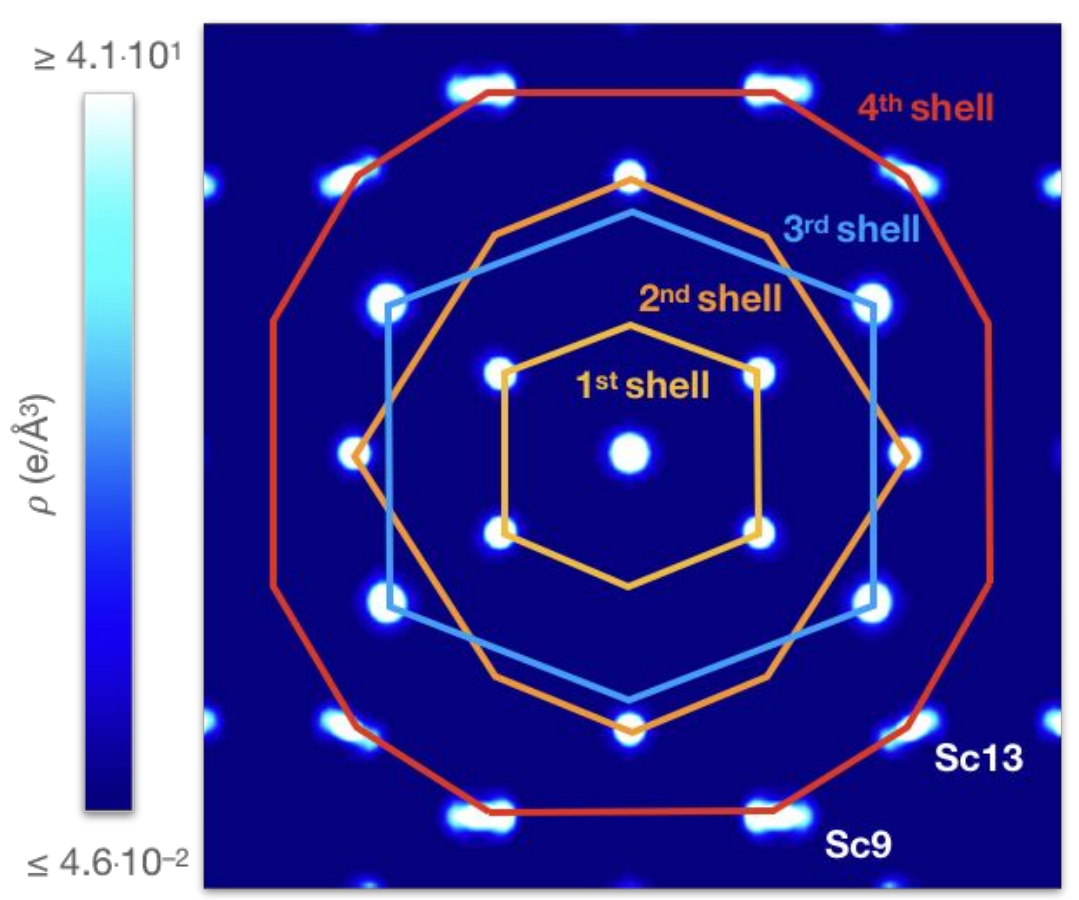

Figure S4. $-0.5<z<0.5$ section of the (001) ED plane overlaid with the edges of the individual shells. The Sc9 and Sc13 atoms showing elongated ADPs and strongly non-spherical ED distributions are labeled exclusively. 
P. Solokha et al. A new 1/1 Mackay type approximant in the Sc-Pd system: from topological data mining to the bench. Supplementary Materials.

Table S3. Comparison between ISAM and MEM standardized structural according to space group Immm (71) Wyckoff symbols, fractional coordinates, site occupancy factor - SOF, isotropic displacement parameters $\left.U_{\text {eq }}\right)$ as well as effective charges $\left(Q_{\text {eff, }}\right.$ determined as atomic number after atom charge subtraction) and Bader volumes for crystal I/ deduced from MEM and DFT.

\begin{tabular}{|c|c|c|c|c|c|c|c|c|c|c|c|c|}
\hline \multicolumn{6}{|l|}{ ISAM } & \multicolumn{5}{|l|}{ MEM } & \multicolumn{2}{|l|}{$D F T$} \\
\hline Atom-Site & $x / a$ & $y / b$ & $z / c$ & SOF $\neq 1$ & $U_{e q}\left(\AA^{2}\right)$ & $x / a$ & $y / b$ & $z / c$ & $Q^{e f f}(e)$ & $\begin{array}{l}\text { Bader } \\
\text { vol. }\left(\AA^{3}\right)\end{array}$ & $Q^{e f f}(e)$ & $\begin{array}{l}\text { Bader } \\
\text { vol. }\left(\AA^{3}\right)\end{array}$ \\
\hline Pd1-2a & 0 & 0 & 0 & & $0.0095(2)$ & 0 & 0 & 0 & -0.8 & 26.3 & -2.8 & 33.89 \\
\hline Pd3-8I & 0 & $0.33396(4)$ & $0.20949(4)$ & & $0.0129(1)$ & 0 & 0.33438 & 0.20985 & -0.6 & 25.8 & -2.6 & 33.74 \\
\hline $\mathrm{Pd} 4-8 m$ & $0.20304(4)$ & 0 & $0.33180(4)$ & & $0.0128(1)$ & 0.20313 & 0 & 0.33188 & -0.9 & 24.5 & -2.9 & 36.30 \\
\hline $\mathrm{Pd} 5-4 j$ & $1 / 2$ & 0 & $0.16162(5)$ & & $0.0149(2)$ & $1 / 2$ & 0 & 0.16204 & $+0.2^{*}$ & $23.3^{*}$ & -2.2 & 31.24 \\
\hline Sc2-4i & 0 & 0 & $0.35401(12)$ & & $0.0100(4)$ & 0 & 0 & 0.35395 & +0.6 & 19.7 & +0.4 & 20.94 \\
\hline Sc3-4e & $0.38047(13)$ & 0 & 0 & & $0.0098(4)$ & 0.38033 & 0 & 0 & +0.7 & 17.7 & +1.3 & 14.46 \\
\hline Sc4-8I & 0 & $0.18179(9)$ & $0.10582(9)$ & & $0.0104(3)$ & 0 & 0.18153 & 0.10594 & +0.3 & 19.8 & +0.7 & 17.41 \\
\hline Sc5-8m & $0.10997(9)$ & 0 & $0.16787(9)$ & & $0.0091(3)$ & 0.10982 & 0 & 0.16799 & +0.2 & 19.5 & -0.5 & 26.75 \\
\hline Sc6-16o & $0.11689(6)$ & $0.17771(6)$ & $0.28750(6)$ & & $0.0104(2)$ & 0.11689 & 0.17811 & 0.28761 & +0.5 & 20.6 & +0.7 & 18.71 \\
\hline Sc7-8I & 0 & $0.32428(10)$ & $0.39604(9)$ & & $0.0123(3)$ & 0 & 0.32385 & 0.39652 & +0.7 & 16.0 & +1.2 & 14.07 \\
\hline Sc8-4g & 0 & $0.37977(14)$ & 0 & & $0.0109(4)$ & 0 & 0.37999 & 0 & +0.4 & 20.8 & +1.0 & 17.49 \\
\hline $\begin{array}{l}\text { Sc9-4f } \\
\text { Pd9-4f }\end{array}$ & $\begin{array}{l}0.1817(11) \\
0.2257(17)\end{array}$ & $\begin{array}{l}1 / 2 \\
1 / 2 \\
\end{array}$ & $\begin{array}{l}0 \\
0\end{array}$ & $\begin{array}{l}0.72(5) \\
0.27(5)\end{array}$ & $\begin{array}{l}0.014(2) \\
0.027(3)\end{array}$ & 0.20241 & $1 / 2$ & 0 & $+4.3^{*}$ & $6.7^{*}$ & +0.1 & 23.09 \\
\hline Sc10-16o & $0.18680(6)$ & $0.30126(7)$ & $0.11099(6)$ & & $0.0110(2)$ & 0.18704 & 0.30153 & 0.11072 & +0.6 & 19.5 & +0.9 & 17.07 \\
\hline Sc11-16o & $0.30213(7)$ & $0.11464(6)$ & $0.17807(6)$ & & $0.0104(2)$ & 0.30219 & 0.11514 & 0.17837 & +0.4 & 19.7 & +1.0 & 16.22 \\
\hline Sc12-8m & $0.39734(10)$ & 0 & $0.33106(10)$ & & $0.0170(3)$ & 0.39755 & 0 & 0.33014 & +0.7 & 16.1 & +0.9 & 15.87 \\
\hline $\begin{array}{l}\text { Sc13-8n } \\
\text { Sc23-8n } \\
\text { Sc33-8n }\end{array}$ & $\begin{array}{l}0.392(3) \\
0.363(2) \\
0.412(5)\end{array}$ & $\begin{array}{l}0.376(2) \\
0.3936(17) \\
0.370(5)\end{array}$ & $\begin{array}{l}0 \\
0 \\
0\end{array}$ & $\begin{array}{l}0.43(3) \\
0.30(3) \\
0.26(3)\end{array}$ & $\begin{array}{l}0.016(7) \\
0.016(3) \\
0.016(7)\end{array}$ & 0.39262 & 0.37679 & 0 & +0.6 & 20.0 & +0.7 & 18.51 \\
\hline
\end{tabular}

*These values reflect the disorder at this site and are not necessarily reasonable.

${ }^{* *}$ The listed values were obtained for the ordered $\mathrm{Sc}_{56} \mathrm{Pd}_{15}$ structure model, with $\mathrm{Sc} 9$ and Sc13 fully occupied (see text) 
P. Solokha et al. A new 1/1 Mackay type approximant in the Sc-Pd system: from topological data mining to the bench. Supplementary Materials.

a)

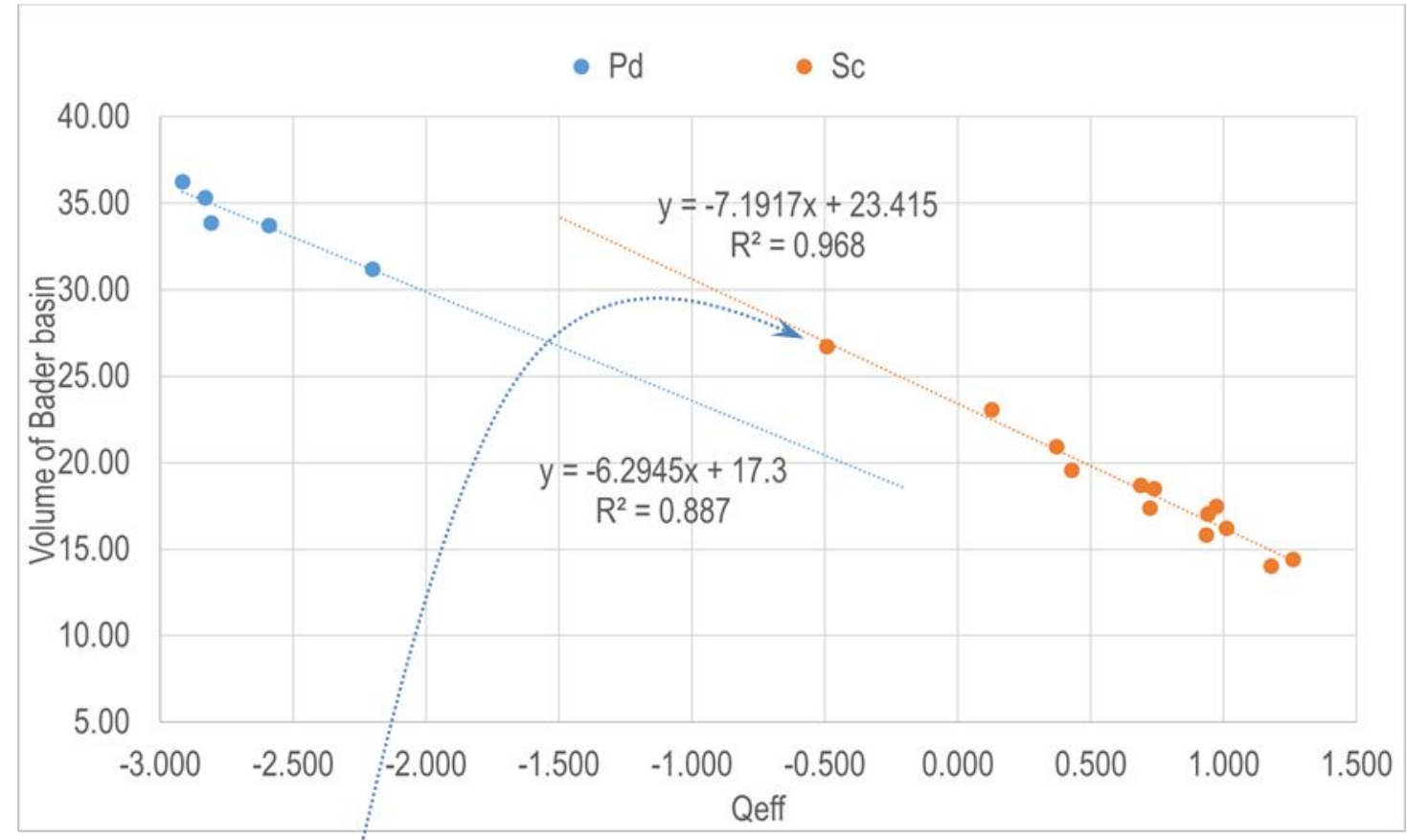

b)

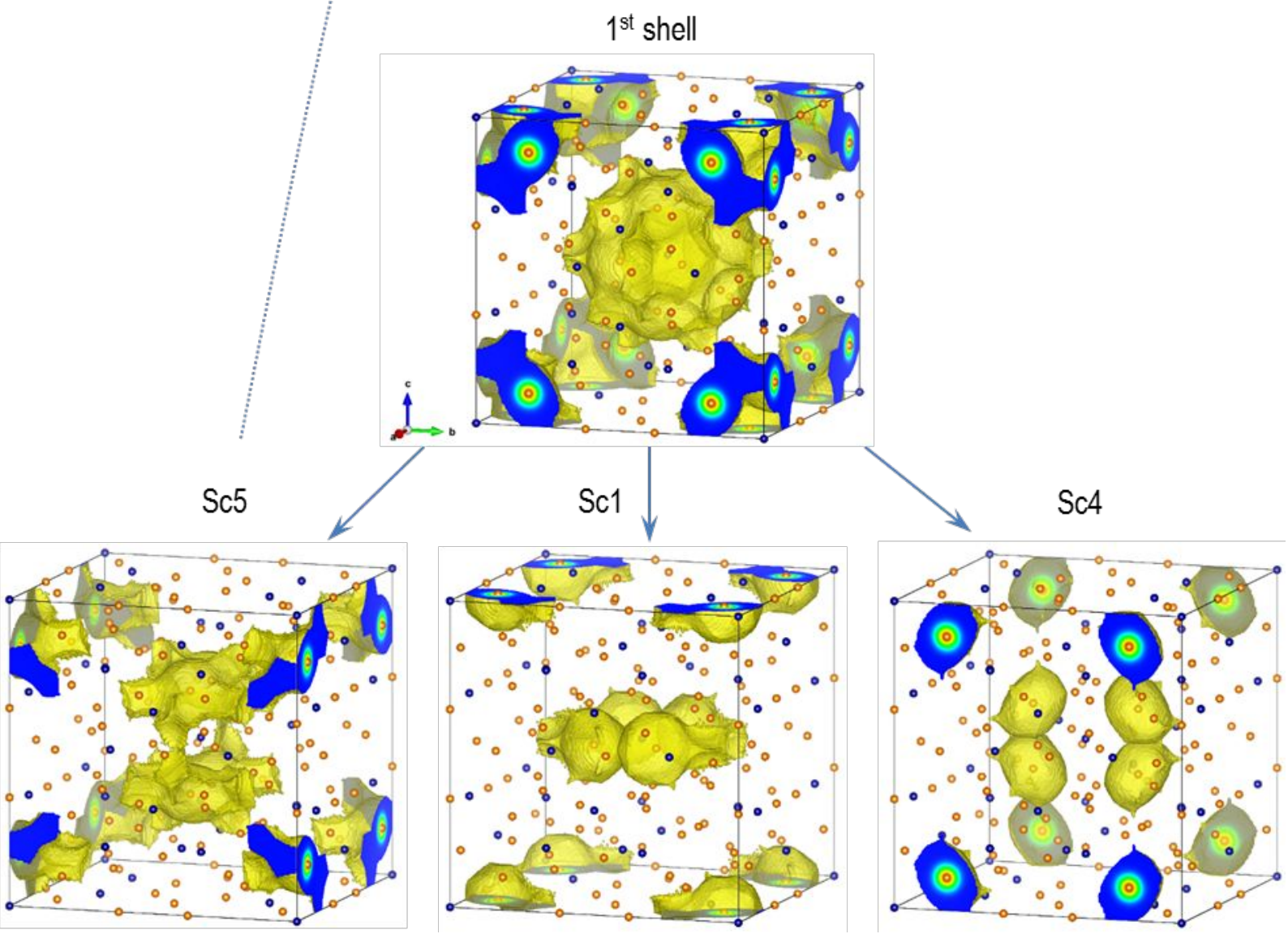

Figure S5. For the $\mathrm{Sc}_{56} \mathrm{Pd}_{15}$ ordered structure modeled by means of DFT: a) Dependencies of Bader volumes vs. $Q_{\text {eff }}$ values with the linear fitting models; b) Bader basins within the $1^{\text {st }}$ icosahedral shell of Mackay cluster and individual Sc5, Sc1 and Sc4 species. 
P. Solokha et al. A new 1/1 Mackay type approximant in the Sc-Pd system: from topological data mining to the bench. Supplementary Materials.

\section{Single and combined defect compositional/configurational space: sampling and energy characteristics}

In order to illustrate the algorithm of the compositional/configurational space sampling, an artificial 2D square structure given in Figure S7, which has two independent sites (two different species) are being disordered, can be considered.

\section{Initial structure}

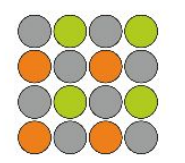

\section{Procedure symbols}

$\square$ Variation of defect concentration/distribution

I. Full configurational space generation

1. Symmetry equivalence filter and optional random selection generation

\section{Single defect compositional/configurational space (SDCCS, $N_{\max }=1$ )}

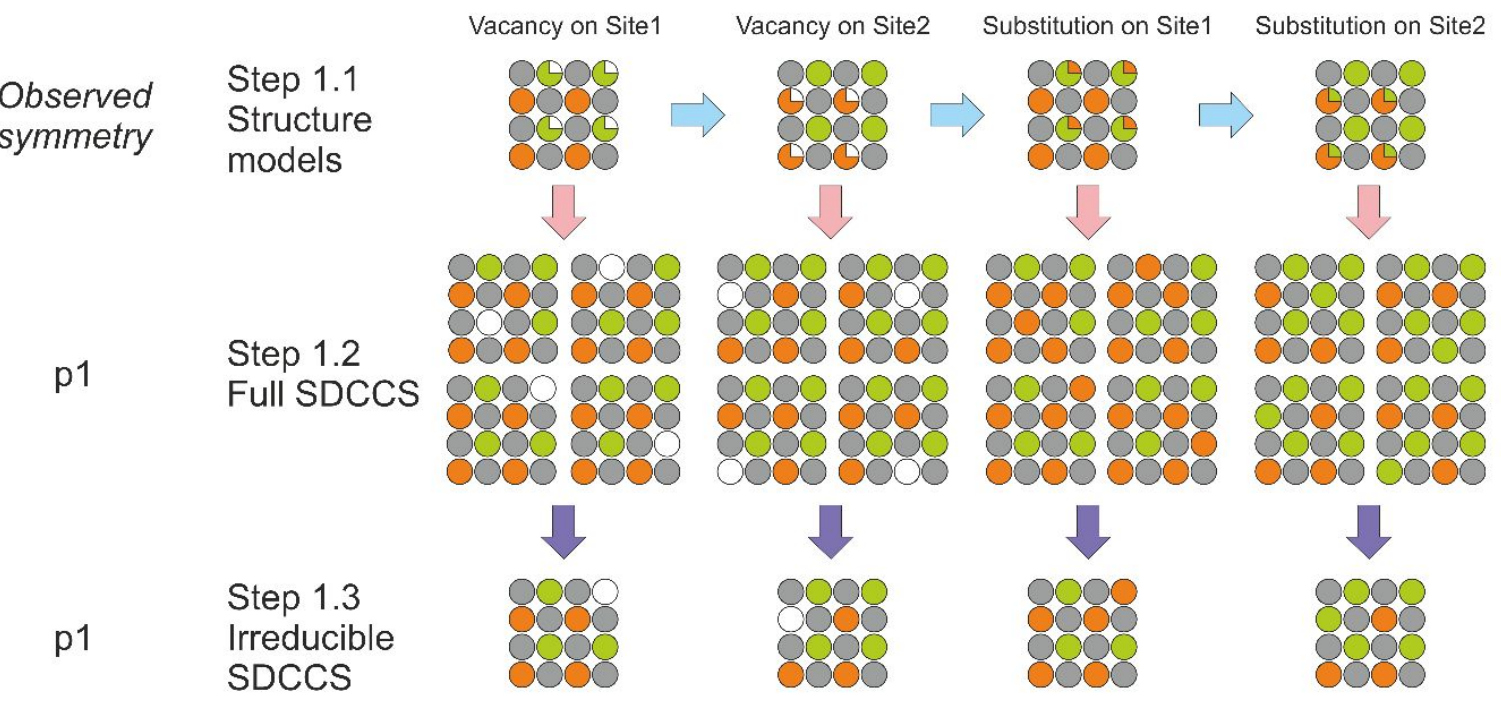

Combined defect compositional/configurational space (CDCCS, $N_{\max }=2$ )

$\begin{array}{llll}\text { Vacancy on Site1 } & \text { Vacancy on Site2 } & \text { Substitution on Site1 } & \text { Substitution on Site2 } \\ \text { Vacancy on Site1 } & \text { Substitution on Site2 } & \text { Substitution on Site2 } & \text { Vacancy on Site1 }\end{array}$

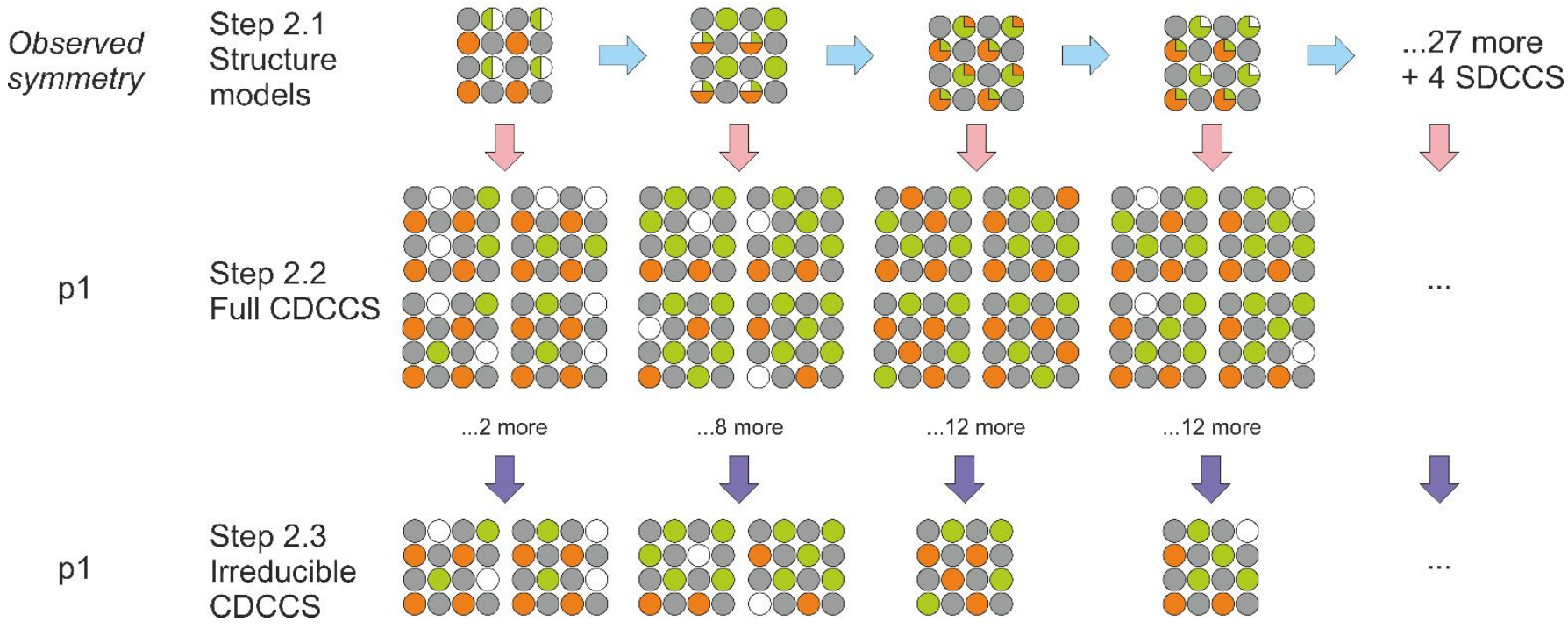

Figure S6. Flowchart of setting single and combined defect compositional/configurational spaces. 
P. Solokha et al. A new 1/1 Mackay type approximant in the Sc-Pd system: from topological data mining to the bench. Supplementary Materials. 
By setting the upper limit of vacancies or substitutions on each site $N_{\max }=1$ (occupancy of $1 / 4$ ), we get 4 different structure models (Figure S7, step 1.1). All possible realizations of each structure model form groups of structure representations containing only one vacancy or only one substituted atom. In turn, these groups constitute full single defect compositional/configurational space (SDCCS) (Figure S7, step 1.2) For the reason of symmetry equivalence of some structure representations within a certain group, the full SDCCS can be reduced to the set of symmetrically inequivalent representations, i.e. irreducible SDCCS (Figure S7, step1.3). Hereafter, the notation 'irreducible' is omitted for short. Obviously, SDCCS of the studied sample structure consist of 4 inequivalent structures.

For the Sc-Pd intermetallic compound studied here, the same approach gives SDCCS consisting of 36 independent entries. For all these structures, the vacancy and substitutional defect formation energies (in terms of the $E_{\text {hull }}$ values) are listed in Table S2.

Table S4. For the studied intermetallic compound, energy properties of the SDCCS entries. The distanced to the central atom of Mackay cluster and the corresponding Wyckoff positions are listed for all independent crystallographic sites.

\begin{tabular}{|c|c|c|c|c|c|c|}
\hline \multirow{2}{*}{ Cluster structure } & \multirow{2}{*}{ Atom } & \multirow{2}{*}{$\begin{array}{c}\text { Distance, } \\
A\end{array}$} & \multirow{2}{*}{$\begin{array}{l}\text { Wyckoff } \\
\text { site }\end{array}$} & \multicolumn{3}{|c|}{$\begin{array}{c}E_{\text {hull, }}, \mathrm{meV} / \text { atom } \\
\left(\mathrm{Sc}_{2} \mathrm{Pd}-\mathrm{Sc}_{56} \mathrm{Pd}_{15}-\mathrm{Sc} \text { hull }\right)\end{array}$} \\
\hline & & & & vacancy & substitution & $\begin{array}{l}\text { mean } \\
\text { value }\end{array}$ \\
\hline Central atom & Pd1 & - & $2 c$ & 13.5751 & 12.6282 & 13.1017 \\
\hline \multirow{3}{*}{$1^{\text {st }}$ shell } & Sc5 & 2.940 & $8 m$ & 12.6250 & 7.7494 & 10.1872 \\
\hline & Sc1 & 2.992 & $8 n$ & 13.4119 & 8.9026 & 11.1573 \\
\hline & Sc4 & 3.043 & 81 & 14.5149 & 9.6445 & 12.0797 \\
\hline \multirow{6}{*}{$2^{\text {nd }}$ shell } & Sc6 & 5.227 & $16 o$ & 13.7917 & 10.2918 & 12.0418 \\
\hline & $\mathrm{Sc} 2$ & 5.229 & $4 i$ & 15.2239 & 10.2243 & 12.7241 \\
\hline & Sc11 & 5.311 & $16 o$ & $1.4029^{*}$ & 8.2271 & $4.8150^{*}$ \\
\hline & Sc10 & 5.345 & $16 o$ & 15.4769 & 10.7156 & 13.0962 \\
\hline & Sc3 & 5.426 & $4 f$ & 18.9595 & 14.6493 & 16.8044 \\
\hline & Sc8 & 5.455 & $4 h$ & 17.4491 & 10.2664 & 13.8577 \\
\hline \multirow{3}{*}{$3^{\text {rd }}$ shell } & $\mathrm{Pd} 2$ & 5.632 & $8 n$ & 4.3125 & 7.8085 & 6.0605 \\
\hline & Pd3 & 5.719 & 81 & 8.0338 & 5.3738 & 6.7038 \\
\hline & $\mathrm{Pd} 4$ & 5.724 & $8 m$ & 4.7193 & 5.8646 & 5.2920 \\
\hline \multirow{5}{*}{$\begin{array}{c}4^{\text {th }} \text { shell (partially due to } \\
\text { the overlapping) }\end{array}$} & Sc7 & 7.479 & 81 & 10.2776 & 7.9874 & 9.1325 \\
\hline & Pd5 & 7.522 & $4 j$ & 3.3377 & 3.9499 & 3.6438 \\
\hline & Sc12 & 7.751 & $8 m$ & 0.6511 & 2.6167 & 1.6339 \\
\hline & Sc13 & 7.754 & $8 n$ & 0.6108 & 8.1899 & 4.4003 \\
\hline & Sc9 & 7.773 & $4 e$ & 0.4935 & -2.1324 & -0.8195 \\
\hline
\end{tabular}

* - the values correspond to the situation when vacancy on Sc11 was occupied by the neighboring Sc atom from Sc12 (see text)

\section{Combined defect compositional/configurational space: sampling and energy properties}

A higher defect concentration, e.g. for $N_{\max }=2$, noticeably complicates a compositional/configurational space sampling. As an example, combination of two point defects requires taking into account a several structure models concerning (see Figure S7, step2.1): (1) combination of a certain defect type (only vacancies or only substitutional defect) on one crystallographic site, (2) contemporary presence of one vacancy and one substitution within the same crystallographic site, (3) the distribution of a certain defect (only vacancies or only substituted atoms) within the different crystallographic positions, (4) distribution of both types of the defects over 
two different positions. The described procedure leads to generation of all possible defect contents and distributions for the combined defect compositional/configurational spaces (CDCCS). Thus, for the sample 2D structure (see Figure S7), total number of structure models is 35 including 4 structure models from SDCCS. The following steps are repeated as for the SDCCS protocol.

Aiming to range the structure models for the intermetallic compound, a Bash-script was used. Within the chosen limits of Sc9 and Sc12 defect concentrations $\left(N_{\max }=4\right), 225$ structure models were generated. By handling all of these models, the Supercell program ${ }^{4}$ sets the same number of configurational spaces $(137,457$ entries of full CDCCS, cf. Figure S7, Step2.2). Subsequently, based on the crystal structure symmetry, the program reduces each group of possible structure representation to that of inequivalent ones (17739 entries of CDCCS, cf. Figure S7 step 2.3).

Additionally, in the case of too complex configurational spaces with many inequivalent structure realizations, an option of random selection of the reduced number of them is also implemented in Superce $/ I^{4}$. By applying this simplification with a maximum number of independent representation equal to 5, the CDCCS was reduced to 1041 entries (relative complexity of 5.87\%). For the selected CDCCS structures, DFT-based relaxation was performed and the obtained $E_{\text {hull }}$ values versus defect concentrations on Sc9 and Sc12 are given in Figure S8.
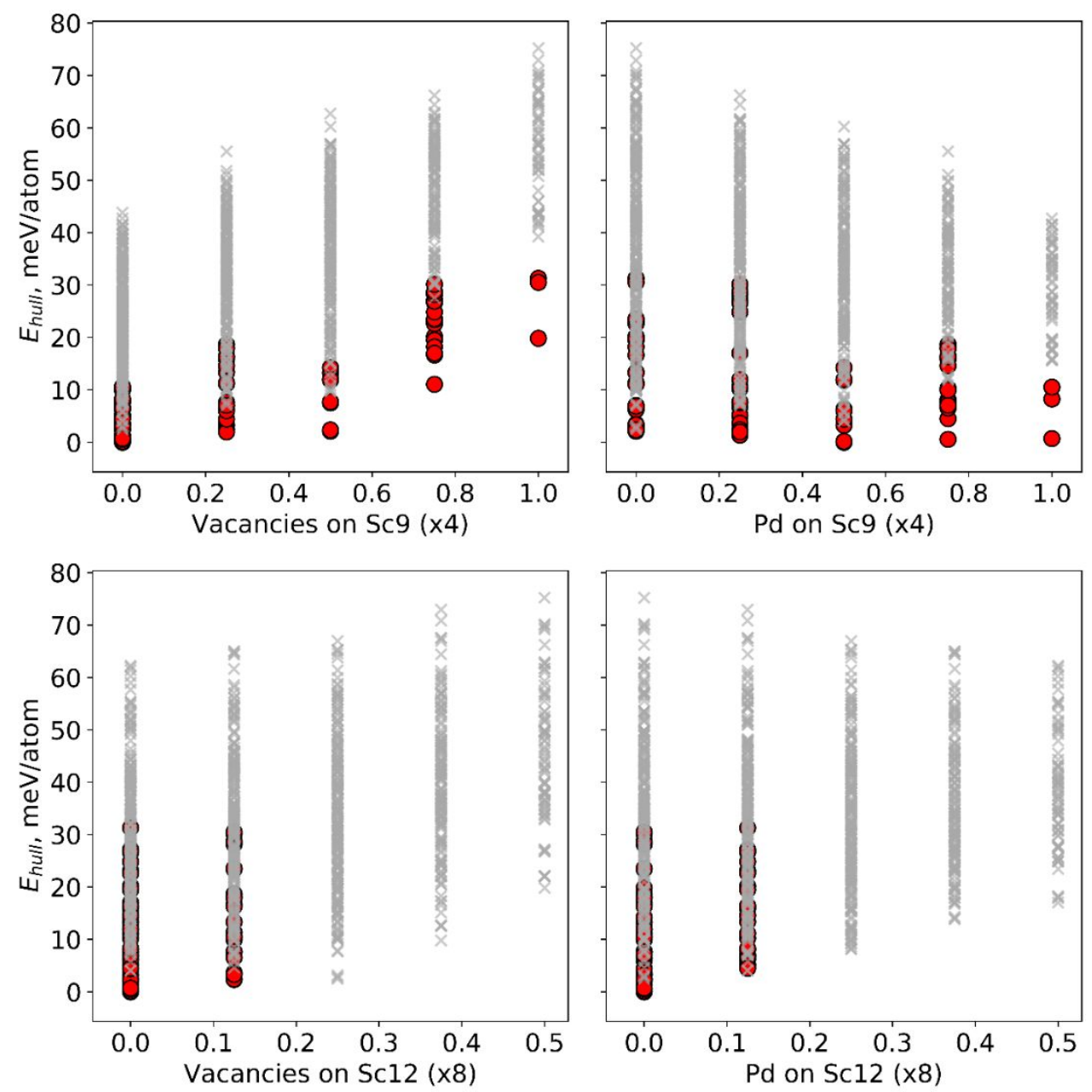

Figure S7. $E_{\text {hull }}$ values in the modeled CDCCS versus concentrations of (a) vacancies on Sc9, (b) $\mathrm{Pd}$ on Sc9, (c) vacancies on Sc12, and (d) Pd on Sc12. The CDCCS entries are divided into two groups: $(i)$ those corresponding to the structural models with less than 5 independent structures and, subsequently, not affected by the random selection stage (red dots) and (ii) the group of randomly reduced configuration sets, whose structure models result in more than 5 independent structure representations (gray crosses). 
Based on the lower limits of the obtained $E_{\text {hull }}$ distributions for different defect levels, one can conclude that (1) the higher concentration of vacancies on both Sc9 and Sc12 as well as the higher $\mathrm{Pd}$ concentration on Sc12 result in higher $E_{\text {hull }}$ limiting values (see Figures S8 a,c, and d, respectively); (2) an increase in the amount of $\mathrm{Pd}$ atoms on Sc9 in the range of 0.0 to 0.5 (from 0 to 2 atoms) leads to a decrease in the $E_{\text {hull }}$ limiting values, whereas the further $\mathrm{Pd}$ content increase (from 2 to 4 atoms) does not change them significantly (see Figure S8 b). These observations are well-correlated with those obtained for SDCCS, within which only the Sc9 antisite defect resulted in the negative $E_{\text {hull }}$ value.

The full sets of the calculated total energies, the formation energies, and the $E_{\text {hull }}$ values corresponding to the $\mathrm{Sc}_{2} \mathrm{Pd}-\mathrm{Sc}_{56} \mathrm{Pd}_{15}$-Sc convex hull are provided inside a SUPPL-Sc56Pd15.xlsx file as two separate sheets according to the influence of the random structure selection stage: the CDCCS part, which is not influenced by the random selection (SUPPL-Sc56Pd15-full), and the rest part of CDCCS (SUPPL-Sc56Pd15-partial).

Table S5. Standardized structural data for DFT relaxed $\mathrm{Sc}_{55} \mathrm{Pd}_{16}: \operatorname{Imm} 2(44), a=14.3716 \AA, b=$ $14.8557 \AA, c=14.2592 \AA$.

\begin{tabular}{lllll|lllll}
\hline Atom & Site & $x / a$ & $y / b$ & $z / c$ & Atom & Site & $x / a$ & $y / b$ & $z / c$ \\
\hline Pd1 & $2 a$ & 0 & 0 & 0.62245 & Sc8 & $8 e$ & 0.11487 & 0.17718 & 0.32164 \\
Pd2 & $4 c$ & 0.20836 & 0 & 0.28788 & Sc9 & $8 e$ & 0.38401 & 0.32345 & 0.42451 \\
Pd3 & $4 c$ & 0.20841 & 0 & 0.9544 & Sc10 & $4 c$ & 0.39298 & 0 & 0.2587 \\
Pd4 & $8 e$ & 0.16566 & 0.29 & 0.12046 & Sc11 & $4 c$ & 0.37263 & 0 & 0.03347 \\
Pd5 & $4 d$ & 0 & 0.32883 & 0.4208 & Sc12 & $4 d$ & 0 & 0.35239 & 0.62314 \\
Pd6 & $4 d$ & 0 & 0.33191 & 0.82284 & Sc13 & $2 a$ & 0 & 0 & 0.24152 \\
Pd7 & $4 d$ & 0 & 0.15958 & 0.11998 & Sc14 & $2 a$ & 0 & 0 & 0 \\
Pd8 & $2 b$ & 0 & $1 / 2$ & 0.94174 & Sc15 & $8 e$ & 0.31565 & 0.39379 & 0.12176 \\
Sc1 & $4 d$ & 0 & 0.32864 & 0.22177 & Sc16 & $4 d$ & 0 & 0.16589 & 0.51436 \\
Sc2 & $4 d$ & 0 & 0.3343 & 0.01371 & Sc17 & $4 d$ & 0 & 0.16922 & 0.73395 \\
Sc3 & $2 b$ & 0 & $1 / 2$ & 0.35462 & Sc18 & $8 e$ & 0.32376 & 0.21278 & 0.00475 \\
Sc4 & $4 c$ & 0.11028 & 0 & 0.4432 & Sc19 & $8 e$ & 0.32192 & 0.2124 & 0.23873 \\
Sc5 & $4 c$ & 0.10859 & 0 & 0.79812 & Sc20 & $8 e$ & 0.17689 & 0.10345 & 0.12375 \\
Sc6 & $8 e$ & 0.30316 & 0.11115 & 0.43534 & Sc21 & $4 c$ & 0.38241 & 0 & 0.61924 \\
Sc7 & $8 e$ & 0.19908 & 0.39016 & 0.30953 & & & & & \\
\hline
\end{tabular}



the bench. Supplementary Materials.
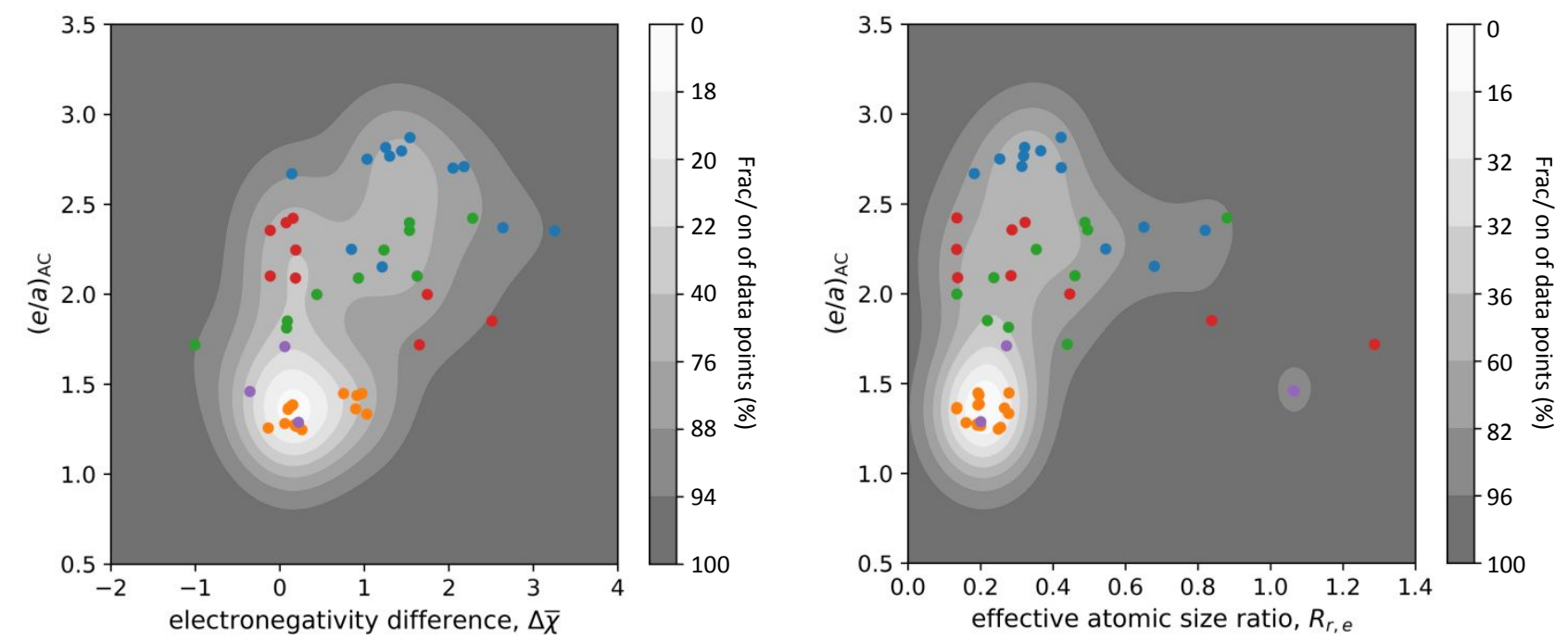

Figure S8. Point density maps representing the selected Mackay-type ACs distribution as a function of different parameters: average number of valence electrons per atom $(\mathrm{e} / \mathrm{a})_{\mathrm{AC}}$, electronegativity difference $\Delta \bar{\chi}$, and effective atomic size $R_{\text {r.e. }}$ The grey-shaded areas represent increasing point densities from dark grey to white. The cumulative percentage of data points is given on the right side of each figure.

\section{References}

(1) Belsky, A.; Hellenbrandt, M.; Karen, V. L.; Luksch, P. New Developments in the Inorganic Crystal Structure Database (ICSD): Accessibility in Support of Materials Research and Design. Acta Crystallogr. Sect. B Struct. Sci. 2002, 58 (3), 364-369. https://doi.org/10.1107/S0108768102006948.

(2) Okamoto, H. Pd-Sc (Palladium-Scandium). J. Phase Equilibria 2002, 23 (6), 554-554. https://doi.org/10.1361/105497102770331334.

(3) Gelato, L. M.; Parthé, E. STRUCTURE TIDY - a Computer Program to Standardize Crystal Structure Data. J. Appl. Crystallogr. 1987, 20 (2), 139-143. https://doi.org/10.1107/S0021889887086965.

(4) Okhotnikov, K.; Charpentier, T.; Cadars, S. Supercell Program: A Combinatorial StructureGeneration Approach for the Local-Level Modeling of Atomic Substitutions and Partial Occupancies in Crystals. J. Cheminform. 2016, 8 (1), 17. https://doi.org/10.1186/s13321-0160129-3. 